\title{
An Ion-Trap Phonon Laser
}

\author{
K Vahala*, M Herrmann, S Knünz, V Batteiger, \\ G Saathoff, T W Hänsch, Th Udem \\ Max Planck Institute für Quantenoptik, 85748 Garching, Germany \\ *Permanent address: California Institute of Technology, Pasadena, California 91125, USA
}

Cooling of atoms and ions $[1,2]$ using a red-detuned laser has had a profound impact on science and technology [3-5]. In this work simultaneous laser cooling and blue-detuned laser pumping of a $\mathrm{Mg}^{+}$ion in a Paul trap is studied. Blue-detuned pumping is conventionally referred to as the heating regime, and in early work, remarkably complex behaviors (bistability and limit cycles) have been associated with this regime [6,7]. These behaviors have so far not been fully explained. Here, it is shown that blue-detuned pumping, as opposed to heating, causes stimulated emission of center-of-mass phonons, leading to coherent oscillatory motion of the ion in analogy with a laser. Mechanical amplification is calculated as well as the threshold pumping condition for oscillation. A single ion in a linear radio-frequency trap is studied to verify these predictions (figure 1 left panel). Blue-detuned pumping of the magnesium D2 transition at $279.6 \mathrm{~nm}$ provides amplification along the long axis of the ion trap so as to excite only axial oscillations. A slightly off-axis, red-detuned beam cools the center-of-mass motion to approximately $1 \mathrm{mK}$. The experimental arrangement is identical to that detailed in ref. [8].

Imaging the ion motion was possible by monitoring luminescence caused by the cooling and amplification beams (Figure 1 right panel). With increasing amplification beam intensity there is a transition to a double-lobed pattern, produced by time averaging of the oscillatory motion of the ion. Further increases in intensity lead to larger amplitudes. Measurements of the threshold and the amplitude of motion versus pumping using such plots are in excellent agreement with the theory. The production of semi-classical, vibrational coherence can be a new tool for study of quantum phenomena using ionic systems, or to drive modes within an effective ionic medium.
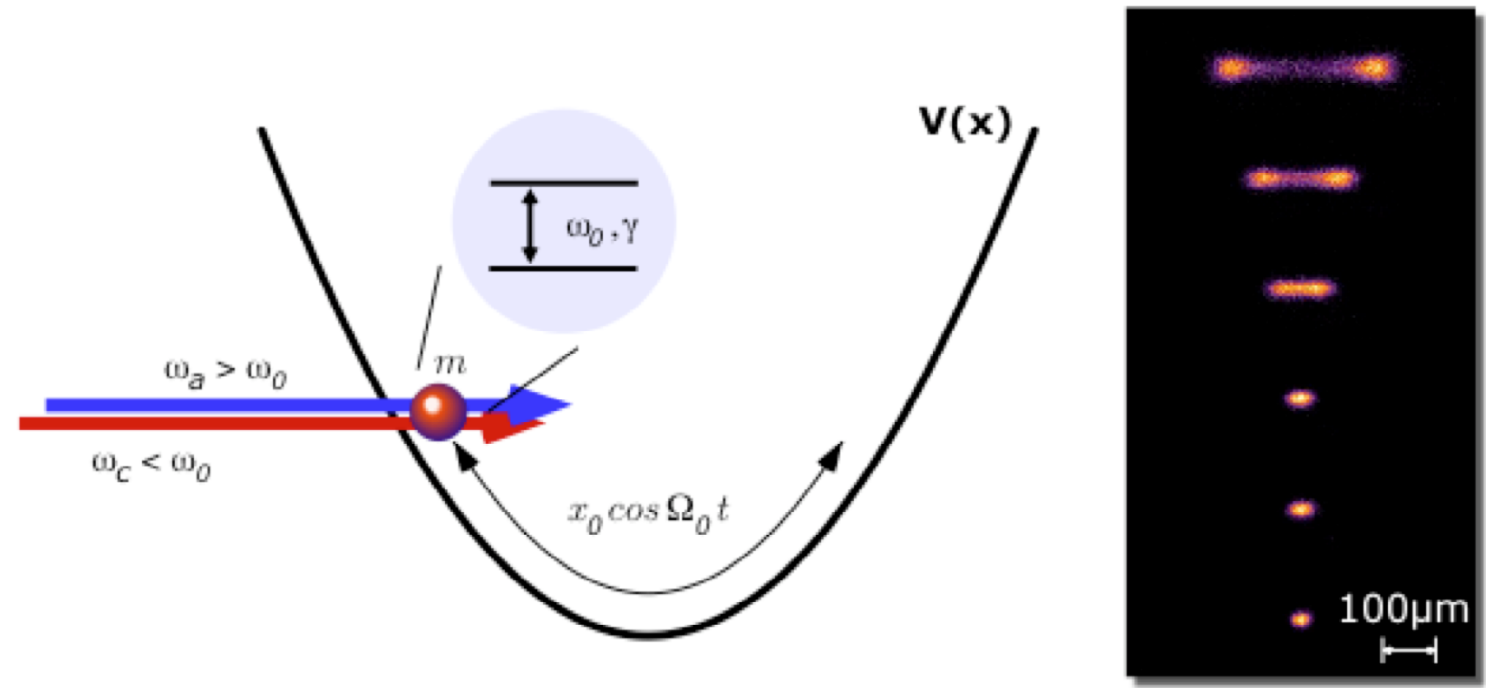

Fig. 1 Left panel: ion in a harmonic trap with secular frequency $\Omega_{\mathrm{o}}$ excited with both a cooling beam at frequency $\omega_{\mathrm{c}}$ and an amplification beam at frequency $\omega_{\mathrm{a}}$ The ion transition (frequency $\omega_{\mathrm{o}}$ ) has linewidth $\gamma$. Right panel: a series of images of ion luminescence in which the amplification beam intensity is increased from lower to upper images. The cooling beam intensity is constant and the lowest image features no amplification. The ion's lateral motion is time averaged, and a threshold is apparent between the third and fourth images.

\section{References}

[1] T.W. Hänsch and A.L.Schawlow, Opt. Communications 13 (1975).

[2] D.Wineland and H. Dehmelt, Bulletin American Physical Society 20 (1975).

[3] R. Blatt and D. Wineland, "Entangled States of Trapped Atomic Ions, ” Nature, 453, 1008 (2008).

[4] K. Helmerson and W.D. Phillips, "Cooling, trapping and manipulation of atoms and Bose-Einstein condensates: Applications to metrology, ” Rivista del Nuovo Cimento, 31, 141 (2008).

[5] I. Bloch, “Quantum Gases, ” Science, 319, 1202 (2008).

[6] W. Quint, “Chaos und Ordnung von lasergekuhlten Ionen in einer Paul-Falle,” Doctoral Thesis, Ph.D. thesis, Ludwig Maximillians Universit, Munich (1990).

[7] T. Sauter, H. Gilhaus, I. Siemers, R. Blatt, W. Neuhauser, P. Toschek, “On the photodynamics of single ions in a trap,” Z. Phys. D, 10, $153-163$ (1988).

[8] M. Herrmann, V. Batteiger, S Knünz, G. Saathoff, Th. Udem, and T. W. Hänsch, "Frequency Metrology on single trapped ions in the weak binding limit: The 3s 1/2-3p3/2 transition in 24-Mg+," Physical Review Letters (in press). 